\title{
The association of smoking and the cost of military training
}

\author{
Robert C Klesges, C Keith Haddock, Cyril F Chang, G Wayne Talcott, Harry A Lando
}

University of

Memphis, Memphis, Tennessee, USA

$\mathrm{R}$ C Klesges

C F Chang

University of Missouri, Kansas City, Missouri, USA

C K Haddock

USAF Surgeon

General's Office,

Washington DC, USA

$\mathrm{G}$ W Talcott

University of

Minnesota,

Minneapolis,

Minnesota, USA

H A Lando

Correspondence to:

Robert C Klesges PhD, The

University of Memphis

Center for Community

Health, Suite 675,5350

Poplar Avenue, Memphis,

TN 38119, USA

bklesges@memphis.edu)

Received 7 June 2000 and in revised form

29 August 2000 . Accepted

12 October 2000

\begin{abstract}
Objective-To determine if premature discharge from the US Air Force was associated with the smoking status of recruits.

Design and setting-A total of 29044 US
\end{abstract}

Air Force personnel recruited from August 1995 to August 1996 were administered baseline behavioural risk assessment surveys during basic military training. They were tracked over a 12 month period to determine those who were prematurely discharged.

Main outcome measures-Excess training costs as a result of premature discharge.

Results- In this 12 month period, $14.0 \%$ of those entering the US Air Force were discharged at a one year follow up. In both univariate and multivariate models, the best single predictor of early discharge was smoking status. Overall, $11.8 \%$ of non-smokers versus $19.4 \%$ of smokers were prematurely discharged (relative risk 1.795$)$.

Conclusions-Using US Department of Defense data on the cost of military training, recruits who smoke in the US Air Force are associated with $\$ 18$ million per year in excess training costs. Applied to all service branches, smoking status, which represents a constellation of underlying behaviours and attitudes that can contribute to early discharge, is associated with over $\$ 130$ million per year in excess training costs.

(Tobacco Control 2001;10:43-47)

Keywords: military; smoking ban; training costs

Smoking is a leading cause of mortality in the USA, accounting for more than 430000 deaths and $30 \%$ of all cancer mortality cases annually. ${ }^{12}$ Smoking also imposes a considerable financial burden on society. In direct medical expenses alone, treatment of smoking related diseases costs about $\$ 50-73$ billion a year or $6-8 \%$ of annual personal health care expenditures. ${ }^{3}$ Smoking has also been linked to disability and job related outcomes, including decreased productivity, increased absenteeism, and longer and more frequent work breaks. ${ }^{4}$

One major potential job related cost that might be associated with smoking is the cost of training individuals for their respective jobs or careers. In this area of smoking research, other than the knowledge that smoking is related to education and that high school dropouts are more likely to be smokers than non-dropouts, ${ }^{6}$ little else is known. This is unfortunate because the USA spends enormous sums of money on employee training, with private employers alone spending $\$ 55.3$ billion in 1995 .

Probably no other organisation spends more time and attention to job training than the US military. Because the length of most military services is four years or less, the US military is constantly training new recruits for a variety of heterogeneous positions, most of which are increasingly technical in nature. Across all service branches, the average cost to train a troop for his/her respective position is $\$ 28800 .^{8}$ This cost includes the cost of basic military training and the specialised training that occurs subsequent to basic training. This latter technical training ranges from a mean of 47 extra days (above and beyond the basic military training) in the US Navy to 82 days in the US Marine Corps.

Given the cost of basic and advanced military training, coupled with the enormous numbers of troops trained every year, the overall cost of training to the US military is nearly $\$ 14$ billion per year. ${ }^{8}$ Of this total, one third is equipment and management costs while fully two thirds are salaries and wages. ${ }^{8}$ As such, anything that significantly impacts the cost of training should be assessed very carefully. The largest excess cost of military training is early discharge - that is, unanticipated release of the troop from military duty during the first year of military service. Discharge in the first year of military service is particularly costly-given that the cost of intensive training occurs-but there is little investigation, if any, of whether smoking is associated with greater likelihood of premature discharge.

Given the strong relation between smoking, health, and job related activities, the purpose of the current investigation is to determine if smoking status is associated with premature discharge in the first year of military training from the US Air Force. Our second purpose is to determine the increased training costs that are associated with smoking status, both in the US Air Force, and extrapolated to other service branches. The findings should provide information useful to the military, as well as the civilian sector, for economising recruitment and training dollars.

\section{Design}

SUBJECTS

Active duty recruits who entered the US Air Force from August 1995 to August 1996 were participants in this study $(\mathrm{n}=29044)$. Mean (SD) age of the respondents was 19.71 (2.05) years. Of all service arms, the US Air Force has the highest level of participation by women, as 
illustrated by a $25.3 \%$ representation of women in this population of recruits in basic military training. The vast majority of the population was single $(75.3 \%)$. Total income for the household in which the recruit was raised was reported for the 12 months before basic military training. Average income for this population was clearly modest with $24.4 \%$ $(\mathrm{n}=7082)$ reporting a total household income lower than $\$ 20000$, and another $50.2 \%$ $(\mathrm{n}=14584)$ reporting a total income of between $\$ 21000$ and $\$ 50000$.

Education level was categorised as "some high school or less but not a diploma, certificate, or GED [general equivalency diploma]" ( $0.2 \%$ of the population); "high school diploma or GED" (63.4\%); "some college or other non-military technical school training" (31.4\%); "two year college degree" $(2.7 \%)$; "four year college degree" (2.0\%); "graduate work but no advanced degree" $(0.2 \%)$; or "advanced degree" $(0.1 \%)$.

The population of US Air Force trainees was ethnically diverse, with $30.2 \%$ from ethnic minorities. Minority breakdown was as follows: African Americans $(14.3 \%, \mathrm{n}=4148)$, Hispanic $(8.5 \%, \mathrm{n}=2468)$, Asian or Pacific Islander $(3.1 \%, \mathrm{n}=906)$, native Americans $(1.0 \%, \quad \mathrm{n}=281)$, and "Other" (3.4\%, $\mathrm{n}=1000)$. Because of their small number in this population, native Americans were included in the "Other" category for these analyses. Human subject protocol approval was obtained from the University of Memphis institutional review board as well as the US Air Force. (The human subjects protocol for this project was reviewed by the Surgeon General's Office CIC as an AFI 40-403 exempt clinical investigation (\#94EX098). The Surgeon General's Office determined this protocol involved the comparison of educational/instructional techniques. As such, it was considered exempt IAW 32 CFR219, 101(b) (1)i.)

PROCEDURE AND MEASURES

Every recruit completed a baseline survey at the start of basic military training, before random assignment to either the smoking intervention or control conditions. The baseline survey was a 53 item behavioural health risk questionnaire. Administration was in a group setting in "flights" (the US Air Force equivalent of platoons) of approximately 50 recruits. Instructions were read and recruits completed all items using a scannable questionnaire. All questionnaires were checked for completeness before the flight departing.

The baseline questionnaire measured four general domains. Firstly, basic demographics were assessed, including height and weight. Secondly, history of tobacco use was assessed. Regular smokers (at least one cigarette per day before basic military training) were asked how many cigarettes they smoked per day in the 12 months before basic military training, and how long they smoked before basic military training. Thirdly, questions thought to be associated with both smoking onset/relapse were queried (for example, US Air Force smoking policies, the percentage of their friends who smoked, rebelliousness, risk taking). Finally, other health risk factors were assessed, including alcohol use, dietary intake, physical activity, and opinions regarding drug use. Because data sets collected on military personnel could be potentially seized or subpoenaed, we did not want to collect data that could be adverse to participants' careers. Opinions regarding drug use, rather than actual behaviour, were queried because admission of any drug use (current or former) is grounds for immediate dismissal in the US military.

Given the numerous quality control checks and the fact that the questionnaire was given as part of basic military training, adherence was extremely high. For the entire sample, there were nearly two million data points collected, with only 10 missing data points. Six week test-retest reliability was obtained on approximately $25 \%$ of the cohort $(n=7080)$. Test-retest correlations averaged 0.73 . Thus, the stability of the responses to this questionnaire was high.

ESTIMATION OF EXCESS DISCHARGES ASSOCIATED WITH SMOKING

To estimate the cost of "excess" early discharges associated with smoking, we first needed the numbers of smokers and non-smokers discharged within the first year of training and the implied relative discharge risks associated with smoking. These numbers can then be used in a simulation model to estimate "excess" discharges under the condition if the discharged smokers had been non-smokers. The early discharge data by smoking status at the one year follow up for the US Air Force are available, and the data collection process is described below. For the other three military branches, only aggregate discharge data are available, requiring that the breakdowns of early discharges by smoking status be estimated.

\section{US Air Force discharge rates.}

This project collaborated with the US Air Force Survey Branch, whose sole mission is to conduct and complete worldwide US Air Force approved surveys. At the one year follow up, participants were located by the world wide locator in the Survey Branch, and their addresses and phone numbers were delivered to the project monthly.

Discharge data for the cohort of US Air Force personnel who participated in the study were obtained from the Survey Branch of the Air Force Personnel Command (AFPC), Texas (Lou Datko, Survey Analyst, Surveys Branch HQ AFPC/DPSAS, personal communication, 12 October 1998). The AFPC database was cross referenced with the smoking cessation programme data set to determine both overall discharge rates and relative risk of discharge caused by smoking. Therefore, the discharge data for the US Air Force personnel reported in this study are based on an actual census of the smoking and discharge status of study participants. 
Table 1 Costs of US military training and its relation to smoking

\begin{tabular}{llllll}
\hline & $\begin{array}{l}\text { Smoking } \\
\text { prevalence (\%) }\end{array}$ & $\begin{array}{l}\text { Number } \\
\text { trained }\end{array}$ & $\begin{array}{l}\text { Discharged in } \\
\text { first year }\end{array}$ & $\begin{array}{l}\text { No. discharged } \\
\text { associated with smoking }\end{array}$ & $\begin{array}{l}\text { Excess cost of } \\
\text { training smokerst }\end{array}$ \\
\hline Air Force & 28.5 & 29044 & $4055(14.0 \%)$ & 629 & $\$ 18115200$ \\
Army & 41.0 & 50799 & $4978(9.8 \%)$ & 1040 & $\$ 29952000$ \\
Navy $^{\star}$ & 39.0 & 51018 & $6938(13.6 \%)$ & 1393 & $\$ 40118400$ \\
Marines $^{\star}$ & 44.7 & 36718 & $6683(18.2 \%)$ & 1494 & $\$ 43027200$ \\
\hline
\end{tabular}

* Assumes identical ratio to data collected in the US Air Force: $11.8 \%$ of non-smokers discharged compared to $19.4 \%$ of smokers.

$\dagger$ The uniform cost of $\$ 28800$ for one year of training across all service branches is used.

Discharge rate for US Army, Navy, and Marines by smoking status.

Smoking prevalence rates for US Army, Navy, and Marines were estimated with data from the most recently published US Department of Defense survey of health related behaviours among military personnel. ${ }^{9}$ This survey is based on a representative sample of all military personnel and provides epidemiological data to the Department of Defense to establish health care policy. The smoking rates for each of the non-Air Force military branches were based on the prevalence of smoking by individuals in the 18-25 age range within each military branch. ${ }^{9}$ These rates are reported in column 1 of table 1 .

We then multiplied these rates by the number of total trainees of the respective military branches (column 2) to derive the numbers of smokers and non-smokers (not shown in table 1). The numbers of smokers and non-smokers, together with the numbers of total discharges in the first year (column 3), provide estimates of the number of discharges associated with smoking status using the following formula for each of the non-Air Force military branches:

Total discharges $=$ number of discharged smokers + number of discharged non-smokers $=1.644 \cdot \mathrm{X} \cdot$ (number of smokers) $+\mathrm{X} \cdot$ (number of non-smokers)

where $\mathrm{X}$ is the discharge rate for non-smokers of a non-Air Force military branch, and 1.644 is the ratio of discharge rates between smokers and non-smokers in the US Air Force (that is, $19.4 \% / 11.8 \%)$. Substituting the numbers of smokers, non-smokers, and total actual discharges for a non-Air Force military branch into the equation, we solve for " $\mathrm{X}$ ". Applying $\mathrm{X}$ to the total number of trainees (column 2), we derived an estimate of the total discharges if no trainee smoked (not shown). The difference between the number of actual discharges (column 3) and the estimated number of discharges if no trainee smoked is the number of "excess" discharged associated with smoking. These are presented in column 4 of table 1 for each of the military branches. Finally, excess training costs associated with smoking are derived by multiplying the excess number of discharges associated with smoking by the average unit cost of basic and technical training across the military branches at $\$ 28800$. $^{8}$

\section{Results}

As can be seen in table 1 , smoking prevalence ranged from a low of $28.5 \%$ (US Air Force) to a high of $44.7 \%$ (US Marines), while the number of troops trained ranged from 29044
(US Air Force) to slightly more than 51000 (US Navy). Discharge rates ranged from a low of $9.8 \%$ (US Army) to a high of $18.2 \%$ (US Marines).

Results on the relation between smoking status and discharge in the US Air Force indicated that the univariate odds ratio (OR) was significant (OR 1.795, 95\% confidence interval (CI) 1.676 to 1.923 ; $\mathrm{p}<0.0001$ ). Overall, smokers were approximately 1.8 times more likely to be discharged in the first year of military service than non-smokers. Of the 14\% discharged in the US Air Force, $19.4 \%$ of smokers were discharged compared to $11.8 \%$ of non-smokers. Several multivariate models were also conducted to determine if other risk factors (for example, demographics, alcohol intake) moderated the relation between smoking status and discharge. The univariate odds ratio between smoking status and discharge changed minimally in these models. When smoking status and all demographic variables (that is, age, sex, ethnic background, family income, educational attainment) and their interactions with smoking status are force entered into a multivariate logistic regression model, the adjusted relative risk between smoking and discharge rises to 5.29 (95\% CI 2.28 to 12.27 ). Thus, to be both conservative and parsimonious, all data are presented based on the univariate relation between smoking and discharge.

Table 1 also presents the number of excess discharges associated with smoking status and the resultant cost. The number of troops discharged associated with smoking ranged from 629 in the US Air Force (which has the lowest smoking prevalence and lowest number trained) to 1494 in the US Marine Corps (which has the highest smoking prevalence rate as well as the highest discharge rate). The resultant excess cost of discharge linked to smoking ranged from slightly more than $\$ 18$ million per year in the US Air Force to $\$ 43$ million per year in the US Marine Corps. Across all service branches, excess training costs associated with smoking were estimated to be $\$ 133660800$ per year or close to $1 \%$ of the total annual military training budget.

\section{Discussion}

The results of the current investigation suggest that smoking is the single best indicator of premature discharge over one year of training from the US Air Force. Excess training costs associated with smoking were approximately $\$ 18$ million per year in the Air Force. Assuming that the same ratio of discharge between smokers and non-smokers applies to other non-Air Force service branches, and that every recruit discharged within the first year would be replaced by another with no benefit derived from the training received by the discharged recruit, total excess training costs related to smoking in the US military were estimated to be over $\$ 130$ million per year.

In civilian populations, smoking is associated with reduced productivity and increased 
absenteeism, ${ }^{10}{ }^{11}$ and it is estimated that smoking among active duty personnel costs the military nearly one billion dollars per year. ${ }^{12}$ Nonetheless, it is surprising that smoking status was the best single predictor of discharge and not demographic factors or even other lifestyle factors, such as attitudes toward drug use or alcohol intake. This finding was limited by the variables we were able to test. Probably the most parsimonious explanation for this overall finding is that contemporary smoking represents a constellation of behaviours and attitudes, not just smoking per se-that is, smokers tend to be from lower income families, have a lower family income, and are associated with a number of lifestyle factors such as disordered dieting and drug and alcohol abuse, poor diets, and decreased physical activity. ${ }^{13-15}$ A number of these factors could, in the absence of smoking status as a predictor variable, be plausibly related to premature discharge. Thus, the high predictive nature of smoking and its relation to premature discharge could be related not only to smoking status but the concomitant lifestyles and demographic characteristics of smokers. Future studies should more carefully assess smoking, smoking related behaviour, and its relation to economic outcomes such as the financial aspects of discharge from the military.

The implications of this study in terms of potential cost savings to the military are extremely complex. At first glance, a recommendation might be to restrict training in the military to non-smokers and smokers who are willing to quit, since our study suggests that this is likely to be associated with over $\$ 130$ million a year of savings. On the other hand, the military continues to struggle with finding enough qualified personnel to recruit in any given year ${ }^{1617}$ and potentially eliminating $25-30 \%$ of the adult population who smoke would make the task even more daunting and potentially more expensive. Further, one could argue that although $88.2 \%$ of non-smokers successfully made it through one year in the military, a full $80.6 \%$ of smokers also successfully completed this year of training.

On balance, probably the most sensible recommendation would be for the military to step up its efforts to encourage non-smokers to join the armed forces as well as to support smoking cessation efforts among those who smoke. Smoking bans in basic military training appear to be an effective policy intervention for smoking cessation, ${ }^{18}$ and extending this ban to their technical training (where smoking relapse occurs $^{18}$ ) seems like a logical next step. The increased use of behavioural interventions for those who smoke, combined with nicotine replacement therapy, also should be promoted, as it has been consistently shown that smoking cessation programmes are not only effective, but cost-effective as well. ${ }^{19}$ Given that the mechanism linking smoking to excess discharge has not been elucidated, it would be premature to suggest that an effective smoking cessation programme would reduce either discharge or training costs. Regardless, smoking cessation has demonstrable individual and social benefits, and the tentative hypothesis that smoking cessation may decrease attrition (for example, via increased fitness or reduced illness) is at least consistent with the results of this study. Future studies should attempt to discover mechanisms linking smoking to excess discharge.

Though there are a number of strengths to the current study, including the first systematic study of premature discharge in the US Air Force, added capacity to evaluate a large number of demographic and lifestyle factors associated with early discharge, and an ethnically diverse sample, there are a number of issues that limit the generalisability of the findings. First, the prospective predictors of discharge could only be directly studied in the US Air Force and the relative costs of smoking in the other service branches could only be estimated. Although it is reasonable to expect the same relation in other military branches, further research is needed. Second, though the current findings are similar to several of those in the civilian population, future research should determine if smoking status prospectively predicts lengthy (and potentially costly) job training programmes. Third, our study used the average training cost that included fixed costs to estimate the total excess training costs, thus resulting in somewhat overstated estimates of cost savings. Future studies should estimate and use the marginal cost of training (that is, the cost of training an extra recruit) with more detailed data from the military. Finally, results may be limited by relying on self reports of smoking status. However, self reports of smoking status are highly reliable and are considered valid for virtually all assessment and most intervention studies. ${ }^{20}$ Nevertheless, future studies using biochemical levels of smoking exposure may provide additional insights into the relation between smoking and discharge from training related activities.

In summary, smoking is related to early discharge from the US military and may cost the Department of Defense over $\$ 130$ million per year or close to $1 \%$ of the total annual training costs. Aggressive methods for promoting smoking cessation in the US military are highly recommended.

This project was supported by a grant (HL-53478) awarded by the National Heart, Lung, and Blood Institute to the University of Memphis, the University of Minnesota, and Wilford Hall Medical Center (RC Klesges, principal investigator). The views expressed in this article are the authors' and do not reflect the official position of US Air Force basic military training, the US Department of Defense, or the US government. Because trainDepartment of Defense, or the US government. Because train-
ing costs and any potential savings associated with military ing costs and any potential savings associated with military
training are important for military readiness, their relevance to training are important for
the US military is high.

1 US Department of Health and Human Services. Best Practices for comprehensive tobacco control programs, August 1999. Atlanta, Georgia: Public Health Service, Center for Disease Control and Prevention, National Center for Chronic Disease Prevention and Health Promotion, Office on Smoking and Health, August 1999. Preprinted, with corrections.

2 Okuyemi KS, Ahluwalia JS, Harris KJ. Pharmacotherapy of smoking cessation. Archive of Family Medicine 2000;9:270-81.

3 Warner KE, Hodgson TA, Carroll CE. Medical costs of smoking in the United States: estimates, their validity, and their implications. Tobacco Control 1999;8:290-300.

4 US Department of Health and Human Services. Smoking and Health in Americas: a report of the Surgeon General, in Collaboration with the Pan American Health Organeral, in Collaboution with the Pan American Healh Organizations: executive sum Center for Chronic Disease Prevention and Health 
Promotion, Office on Smoking and Health, 1992. (DHHS publication No. (CDC) 92-8421.

5 Helyer AJ, Brehm WT, Perino, L. Economic of tobacco us for the Depar Med 1998;163:217 21 .

6 US Department of Health and Human Services. Preventing tobacco use among young people. A report of the Surgeon General. Atlanta, Georgia: Department of Health and Human Services, Public Health Service, Center for Disease Control and Prevention, National Center for Chronic Disease Prevention and Health Promotion, Office of Smoking and Health, 1994. (US Government Printing Office No. S/N 017-001-00491-0.)

7 Benson G. How much do employers spend on training? Training and Development 1996;50:56-8.

8 Defense Manpower Data Center. Department of Defense institutional training resource trends, fiscal year 1999. Report institutional training resource trends, fiscal year 1999. Report prepared 1998 .

9 Bray RM, Kroutil LA, Wheeless SC, et al. 1995 Department of Defense survey of health related behaviors among military personnel. Report prepared for the Assistant Secretary of

10 Single E, Robson L, Xie X, et al. The economic costs of alcohol, tobacco, and illicit drugs in Canada, 1992. Addiction 1998;93:991-1006.

11 Zheng D, Wheeler FC, Jones PJ, et al. Health and economic impact of cigarette smoking in South Carolina. $\mathcal{F} S \mathrm{C} \mathrm{Med}$ Assoc 1998;94:101-4

12 Department of Defense. Economic consequences of tobacco use for Department of Defense. Unpublished technical report, US Department of Defense; 1996.
13 Gritz ER, Crane LA. Use of diet pills and amphetamines to lose weight among smoking and nonsmoking high school seniors. Health Psychol 1991;10:330-5.

14 Klesges RC, Ward KD, Ray JAW, et al. The prospective relationships between smoking and weight in a young, biracial cohort: the CARDIA study. $f$ Consult Clin Psychol (in press)

15 US Department of Health and Human Services. The health benefits of smoking cessation. A report of the Surgeon General, 1990. Rockville, Maryland: Public Health Service, Center for Disease Control and Prevention, Office on Smoking and Health, 1990. (DHHS Publication No. (CDC) 90-8416.)

16 Komarow S. As Iraq looms, shortfalls hastening "downward slide." USA Today. November 10, 1998:1-2.

17 US Department of Defense. Fiscal year 1998 recruiting achieves $97 \%$ of goal for quantity, while exceeding quality benchmarks. Department of Defense press release 55998; 28 October 1998.

18 Klesges RC, Stone E, Clements M, et al. Smoking cessation in the armed forces: The US Air Force/Universities of Memphis/Minnesota smoking cessation program. Ann Behav Med 1998;20:S29.

19 Klesges RC, Ward KD, DeBon M. Smoking cessation: a successful behavioral/pharmacologic interface. Clin Psychol Rev 1996;16:479-96.

20 Velicer WF, Prochaska JO, Rossi JS, et al. Assessing outcome in smoking cessation studies. Psychol Bull 1992;111:23-41.

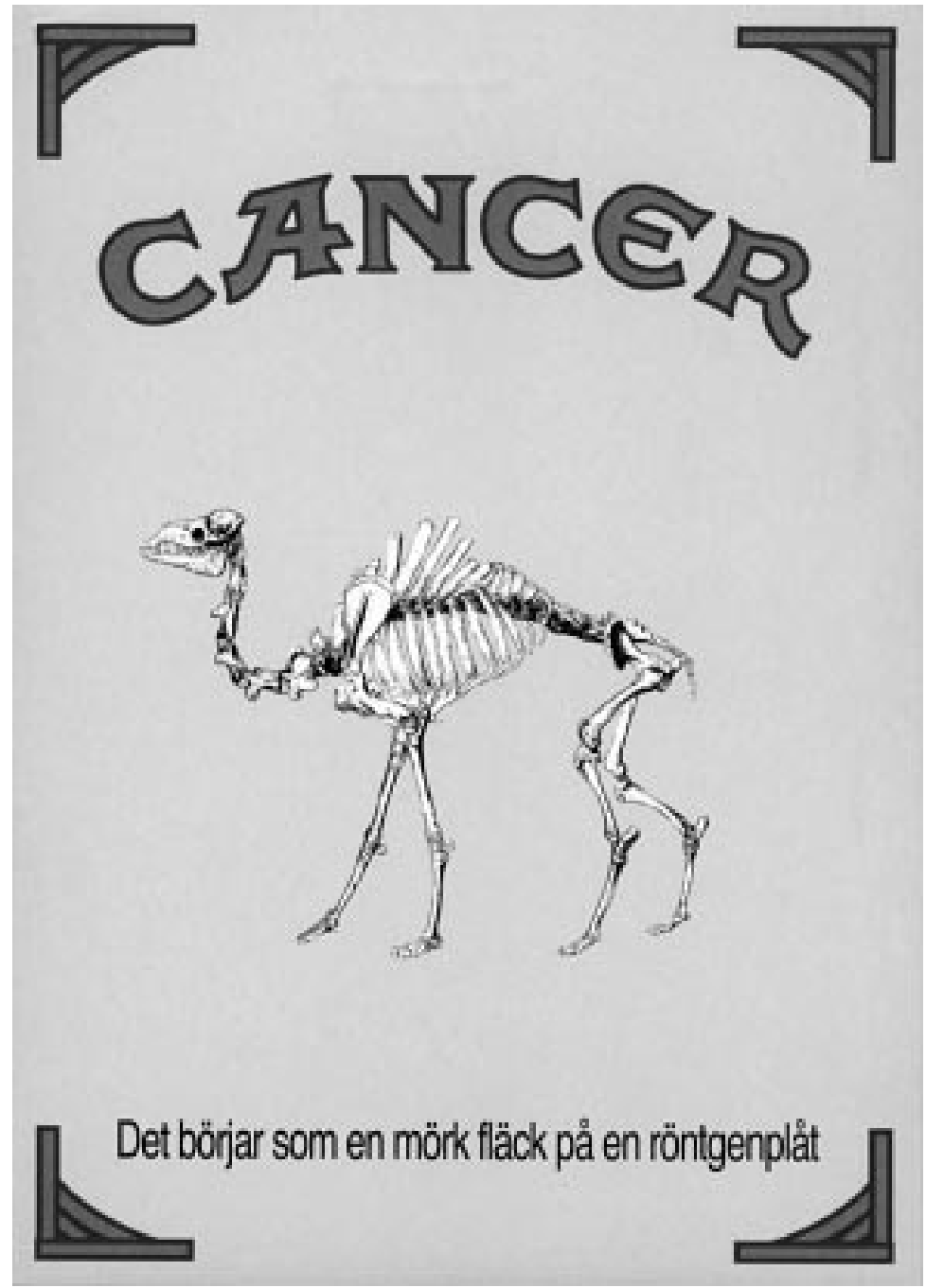

It starts with a dark spot on an $x$ ray. Contributed by Melanie Wakefield. 